\title{
EFECTO DE LA CONGELACIÓN EN LOS PARÁMETROS MORFOMÉTRICOS DEL ESPERMATOZOIDE DE CARNERO
}

\author{
Effect of freezing on the ram sperm morphometric parameters
}

\author{
Wilber Choquepuma ${ }^{1}$, César Ordoñez ${ }^{2}$, Hurley Quispe ${ }^{2}$, Hernán Cucho²
}

1 Escuela Profesional de Ingeniería Agropecuaria, Universidad Nacional de San Antonio Abad del Cusco, Perú

2 2Escuela Profesional de Zootecnia, Universidad Nacional de San Antonio Abad del Cusco, Perú

* Autor correspondiente: Hernan Cucho, Facultad de Ciencias Agrarias, Granja K'ayra, San Jerónimo, Cusco

E-mail address: hernan.cucho@unsaac.edu.pe

Recibido: 09/07/2017

Evaluado: $25 / 07 / 2017$

Aceptado: 13/08/2017

\section{RESUMEN}

El objetivo del estudio fue evaluar el efecto de la congelación en los parámetros morfométricos de los espermatozoides del carnero. Para ello se utilizó el semen de 2 carneros jovenes de la raza Corriedale de 2 y 2.5 años, con una condición corporal de 3.5. Se evaluaron el volumen, motilidad, concentración, viabilidad, integridad de la membrana y la morfometría de los espermatozoides en las muestras. Todas las variables morfometricas se determinaron con un sistema CASA (ISAS, Proiser). El proceso de congelación se realizó con diluyente a base TRIS con $3 \%$ de lecitina de soya y glicerol como crioprotector. Se analizaron 12 parámetros morfométricos del espermatozoide, longitud, anchura, área, perímetro, elipticidad, elongación, regularidad y rugosidad de la cabeza del espermatozoide, porcentaje de acrosoma, y la anchura, distancia y ángulo de inserción de la pieza intermedia del espermatozoide de carnero. Se evaluaron una población de 2518 espermatozoides en semen fresco, refrigerado y descongelado. Nuestros resultados indican que algunos parámetros morfométricos del espermatozoide (longitud, anchura, área y perímetro de la cabeza) criopreservado son de menor dimensión $(p<0.05)$ a lo encontrado en semen fresco y refrigerado.

Palabras clave: CASA, morfometría espermática, criopreservación, semen carnero

\section{ABSTRACT}

The objective of the study was to evaluate the effect of freezing on the morphometric parameters of ram spermatozoa. For this purpose, the semen of 2 young rams of the Corriedale breed of 2 and 2.5 years old was used, with a body condition of 3.5. The volume, motility, concentration, viability, membrane integrity and sperm morphometry were evaluated in the samples. All morphometric variables were determined using a CASA system (ISAS, Proiser). The freezing process was performed with TRIS based diluent with $3 \%$ soy lecithin and glycerol as cryoprotectant. A total of 12 morphometric parameters were analyzed (length, with, area, perimeter, ellipticity, elongation, regularity and roughness of head spermatozoa, acrosome percentage, and the width, distance and insertion angle mid piece ram sperm). A population of 2518 spermatozoa was evaluated in fresh, refrigerated and thawed semen. Our results indicate that some cryopreserved sperm morphometric parameters (length, width, area and perimeter of the head) are smaller $(p<0.05)$ than that found in fresh and refrigerated semen Keywords: CASA, sperm morphometry, cryopreservation, ram sperm

Keywords: CASA, sperm morphometry, cryopreservation, ram sperm 


\section{INTRODUCCION}

La inseminación artificial es la técnica de reproducción asistida más importante para facilitar el progreso genético en las especies de animales domésticas, es por ello su importancia el determinar la potencial fértil de un macho, evaluando su calidad espermática (Maroto, 2012).

El espermiograma convencional utilizado para evaluar una muestra de semen incluye la evaluación del volumen concentración espermática, motilidad, viabilidad, morfología, entre otros. Algunos de estos parametros, tiene el inconveniente de que se realiza de forma subjetiva, limitando la información sobre el potencial fértil de una muestra seminal (Maroto, 2012). Los avances tecnológicos realizados han permitido valorar las características espermáticas de forma objetiva, así se han desarrollado sistemas automáticos de análisis de semen (CASA) (Verstegen et al., 2002).

La morfología espermática es parte esencial de una evaluación seminal, puesto que las muestras con alta proporción de espermatozoides anormales presentan una baja fertilidad (Hafez y Hafez, 2004) y ésta es evaluada muchas veces de forma subjetiva. Sin embargo, este método ha demostrado ser dudoso (Gravance et al., 1997), cuando las anormalidades del espermatozoide no son tan evidentes como para valorarlas de forma visual, es por ello emplear otro tipo de sistemas como por ejemplo los sistemas CASA, permitiran proveer mediciones más objetivas y confiables de estas características (Verstegen et al. 2002).

Maroto (2012), manifiesta que en los estudios realizados para evaluar el efecto del proceso de criopreservación sobre la forma de la cabeza del espermatozoide se ha demostrado una reducción de las dimensiones de la misma tras la descongelación. Las diferencias en el tamaño de la cabeza entre los espermatozoides vivos y muertos posiblemente se deben a que estos últimos se hidratan por los componentes del medio, como consecuencia de la perdida de la permeabilidad de la membrana (Marco-Jiménez et al., 2006.)

Gomedio y Roldan (2008), manifiesta que espermatozoides con cabezas más pequeñas y elongadas nadan más rápido teniendo así más posibilidades de fecundar el ovocito. Se cree también que las modificaciones en la forma y dimensión de la cabeza espermática puedan reflejar defectos en la estructura e integridad de la cromatina del espermatozoide (NúñezMartínez et al., 2005).

La información respecto al efecto de la criopreservación en los parámetros morfométricos del espermatozoide de carnero son escasos (Martí et al., 2011 ; Ramón et al., 2013). Por lo tanto, el presente trabajo, tiene como objetivo evaluar el efecto de la congelación en los parámetros morfométricos de los espermatozoides del carnero.

\section{MATERIALES Y MÉTODOS}

\section{Código de ética}

Los autores declaran que el presente estudio se ha llevado a cabo de acuerdo con el Código de Ética para los experimentos con animales, tal y como se refleja en la normativa: http://ec.europa.eu/environment/chemicals/lab_animals/legisl ation_en.htm.

\section{Zona de estudio $y$ animales}

El estudio se realizó en el Centro de Investigación en Camélidos Sudamericanos (CICAS) La Raya, Facultad de Ciencias Agrarias de la Universidad Nacional de San Antonio Abad del Cusco, ubicada en la provincia de Canchis en las coordenadas $14^{\circ} 28^{\prime} 27.01$ " S y $71^{\circ} 02^{\prime} 45.40^{\prime \prime} \mathrm{W}$ a una altura de 4130 metros de altitud, el trabajo se realizó entre febrero y agosto del 2015.

Se utilizó 2 carneros jóvenes de la raza Corriedale de 2 y 2.5 años de edad, con un peso promedio de $72.50 \mathrm{~kg}$, y $3.5 \mathrm{de}$ condición corporal, libres de parásitos, con simetría testicular, exento de anormalidades físicas y libre de lesiones en prepucio y pene. Los animales se alimentaron a base de rye grass inglés y trébol blanco, además de pasto nativo, con agua a libre disposición.

\section{Colección y tratamiento del semen}

Los carneros fueron sometidos a un período de entrenamiento de 45 días, tras ello se obtuvo 6 eyaculados de cada uno de éstos, con un intervalo de 5 días. El semen se colecto empleando una vagina artificial (Minitube $\AA$ ) para ovinos, termorregulada a $37^{\circ} \mathrm{C}$, empleando una hembra con celo inducido como estímulo. El volumen del eyaculado se determinó por observación directa en un tubo colector graduado $(\mathrm{mL})$. Se determinó la motilidad y concentración espermática empleando $5 \mu \mathrm{L}$ de semen, los que fueron evaluados a 100X aumentos, se empleó el kit Vital Test® para determinar el porcentaje de viabilidad; para la integridad funcional de la membrana (HOST) se utilizó una solución hipoosmótica de $100 \mathrm{mOsm} / \mathrm{L}$. Los análisis de la motilidad, concentración, viabilidad, integridad funcional de la membrana y la morfometría del espermatozoide fue realizado usando el

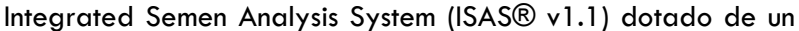
microscopio UOP-UB200i (Proiser R+D, Paterna, Valencia, España).

Para la morfometría espermática, se realizaron frotis empleando $5 \mu \mathrm{L}$ de muestra, una vez secos se tiñeron con Hemacolor® (Merck, Darmstadt, Alemania), según protocolo del producto. La morfometría se evaluó con el módulo de morfología del ISAS $®$, empleando un objetivo de $100 \mathrm{X}$ de campo claro; la resolución del análisis fue de $0.084 \mu \mathrm{m} /$ pixel en ambos ejes, se realizó este análisis de la segunda y quinta colecta de cada animal, las imágenes de no menos de 200 espermatozoides de cada muestra de semen fresco, refrigerado y descongelado fueron capturadas y analizadas.

Se analizarón 12 parámetros morfométricos, de la cabeza del espermatozoide, la longitud $(L, \mu \mathrm{m})$, anchura $(W, \mu \mathrm{m})$, área $(A$, $\mu \mathrm{m} 2)$ y perímetro $(P, \mu \mathrm{m})$, elipticidad $(\mathrm{L} / \mathrm{W})$, elongación ([L$\mathrm{W}] /[\mathrm{L}+\mathrm{W}])$, regularidad $(\pi \mathrm{LW} / 4 \mathrm{~A})$ y rugosidad $(4 \pi \mathrm{A} / \mathrm{P} 2)$ y acrosoma (\% del total de la cabeza); y de la pieza intermedia del espermatozoide la anchura $(\mu \mathrm{m})$, distancia de inserción (distancia entre la cabeza y el eje de la pieza intermedia, $\mu \mathrm{m}$ ) y ángulo de inserción ( $\left.{ }^{\circ}\right)$.

Para la dilución y criopreservación del eyaculado, se utilizó un dilutor base TRIS con lecitina de soya al $3 \%$ (TRIS - $3.25 \mathrm{~g}$, Dfructuosa $-0.93 \mathrm{~g}$, ácido cítrico $-1.70 \mathrm{~g}$, glicerol $-2 \mathrm{~mL}$, lecitina de soya - 3mL, agua bidestilada - hasta $100 \mathrm{ml}$ ) (Aguilar et al., 2013), para homogenizar los componentes de este diluyente se empleó un homogenizador automático a $2500 \mathrm{rpm}$ durante 1 minuto, para luego dejar la solución en baño maría durante 10 minutos a $37^{\circ} \mathrm{C}$. Posteriormente se volvió a homogenizar durante 1 minuto, tras lo cual se centrifugó a $4000 \mathrm{rpm}$ durante 20 minutos, finalmente se extrajo la parte intermedia de las 3 capas que se formaron después del centrifugado Se adicionó la cantidad necesaria de dilutor para lograr una concentración final de $50 \times 10^{6}$ espermatozoides $/ \mathrm{mL}$; se empleó pajuelas (Minitube ${ }^{\circledR}$ ) de $0.5 \mathrm{~mL}$ para la criopreservación del semen. 
Se empleó la estadística descriptiva para evaluar la motilidad, concentración, viabilidad e integridad funcional de la membrana. Para los parámetros morfométricos se determinó la normalidad de las variables en estudio (test de KolmogorovSmirnov), se transformó los datos (proc transreg) de las variables que no mostraban distribución normal y se analizaron en un DCA; para comparar las medias se usó la prueba de Duncan con una significancia de $p<0.05$. Se empleó el SAS en los análisis estadísticos.

\section{RESULTADOS}

El volumen, concentración, motilidad total, viabilidad e integridad de la membrana del semen fresco colectado de los 2 carneros, en seis repeticiones por macho, se muestra en la tabla 1 .

Tabla 1. Características del semen colectado de carneros (promedio, desviación estándar (DE), coeficiente de variación, mínimos y máximos). n: colecta; DE: desviación estándar; CV: coeficiente de variación; Min: mínimo; Max: máximo

\begin{tabular}{|c|c|c|c|c|c|c|}
\hline Variable & $\mathrm{n}$ & Media & $\mathrm{DE}$ & $\% \mathrm{CV}$ & Min & $\operatorname{Max}$ \\
\hline $\begin{array}{l}\text { Volumen (ml) } \\
\text { Concentración }\end{array}$ & 12 & 1.03 & 0.53 & 12.61 & 0.80 & 1.20 \\
\hline (106 spz/ml) & 12 & 2591.9 & 203.7 & 7.8 & 2208.1 & 2835.8 \\
\hline $\begin{array}{l}\text { Motilidad } \\
\text { total (\%) } \\
\text { Viabilidad }\end{array}$ & 12 & 86.60 & 3.37 & 3.90 & 82.14 & 91.13 \\
\hline (\%) & 12 & 86.13 & 2.11 & 2.45 & 83.59 & 90.84 \\
\hline HOST (\%) & 12 & 80.76 & 2.02 & 2.50 & 77.45 & 83.50 \\
\hline
\end{tabular}

Tabla 2. Valores (promedio $\pm \mathrm{DE}$ ) de los parámetros morfométricos de los espermatozoides de carneros en fresco, refrigerado y descongelado. $n$ : espermatozoides; DE: desviación estándar. Letras diferentes en la horizontal muestran diferencias significativas $(\mathrm{p}<0.05)$

\begin{tabular}{lccc}
\hline \multicolumn{1}{c}{ Variable } & Fresco & Refrigerado & Descongelado \\
\hline \multicolumn{1}{c}{$\mathrm{n}$} & 839 & 839 & 840 \\
Longitud cabeza $(\mu \mathrm{m})$ & $8.52 \pm 0.37 \mathrm{a}$ & $8.52 \pm 0.38 \mathrm{a}$ & $8.34 \pm 0.34 \mathrm{~b}$ \\
Anchura cabeza $(\mu \mathrm{m})$ & $4.64 \pm 0.18 \mathrm{a}$ & $4.60 \pm 0.18 \mathrm{~b}$ & $4.61 \pm 0.17 \mathrm{~b}$ \\
Área cabeza $\left(\mu \mathrm{m}^{2}\right)$ & $32.11 \pm 1.52 \mathrm{a}$ & $32.05 \pm 1.51 \mathrm{a}$ & $31.92 \pm 1.46 \mathrm{~b}$ \\
Perímetro cabeza $(\mu \mathrm{m})$ & $24.34 \pm 1.01 \mathrm{a}$ & $24.42 \pm 1.27 \mathrm{a}$ & $24.23 \pm 1.10 \mathrm{~b}$ \\
Acrosoma $(\%$ cabeza) & $50.38 \pm 7.53 \mathrm{a}$ & $48.40 \pm 8.10 \mathrm{a}$ & $43.97 \pm 8.39 \mathrm{~b}$ \\
Elipticidad & $1.84 \pm 0.10 \mathrm{a}$ & $1.85 \pm 0.10 \mathrm{a}$ & $1.81 \pm 0.09 \mathrm{~b}$ \\
Rugosidad & $0.68 \pm 0.04 \mathrm{a}$ & $0.68 \pm 0.04 \mathrm{a}$ & $0.69 \pm 0.05 \mathrm{a}$ \\
Elongación & $0.29 \pm 0.02 \mathrm{a}$ & $0.31 \pm 0.03 \mathrm{a}$ & $0.29 \pm 0.02 \mathrm{a}$ \\
Regularidad & $0.97 \pm 0.03 \mathrm{a}$ & $0.96 \pm 0.03 \mathrm{a}$ & $0.95 \pm 0.03 \mathrm{a}$ \\
Anchura pieza intermedia $(\mu \mathrm{m})$ & $1.47 \pm 0.76 \mathrm{a}$ & $1.35 \pm 0.76 \mathrm{~b}$ & $1.44 \pm 0.80 \mathrm{a}$ \\
Distancia pieza intermedia $(\mu \mathrm{m})$ & $0.14 \pm 0.10 \mathrm{a}$ & $0.14 \pm 0.12 \mathrm{a}$ & $0.14 \pm 0.10 \mathrm{a}$ \\
Ángulo pieza intermedia $\left({ }^{\circ}\right)$ & $4.39 \pm 3.29 \mathrm{~b}$ & $4.54 \pm 3.19 \mathrm{~b}$ & $4.77 \pm 3.19 \mathrm{~b}$ \\
\hline
\end{tabular}

Después de la descongelación de semen, se obtuvo una motilidad total de $45.69 \pm 2.19 \%$, una viabilidad de $45.09 \pm$ $2.84 \%$, y una integridad funcional de la membrana (HOST) de calidad de semen congelado.

Se han evaluado 12 parámetros morfométricos del espermatozoide de carnero (Figura 1) (Tabla 2); se han encontrado diferencias significativas $(p<0.05)$ para el longitud, anchura, área y perímetro de la cabeza del espermatozoide de los carneros tras ser descongelados, en relación con sus medidas en fresco y refrigerado.

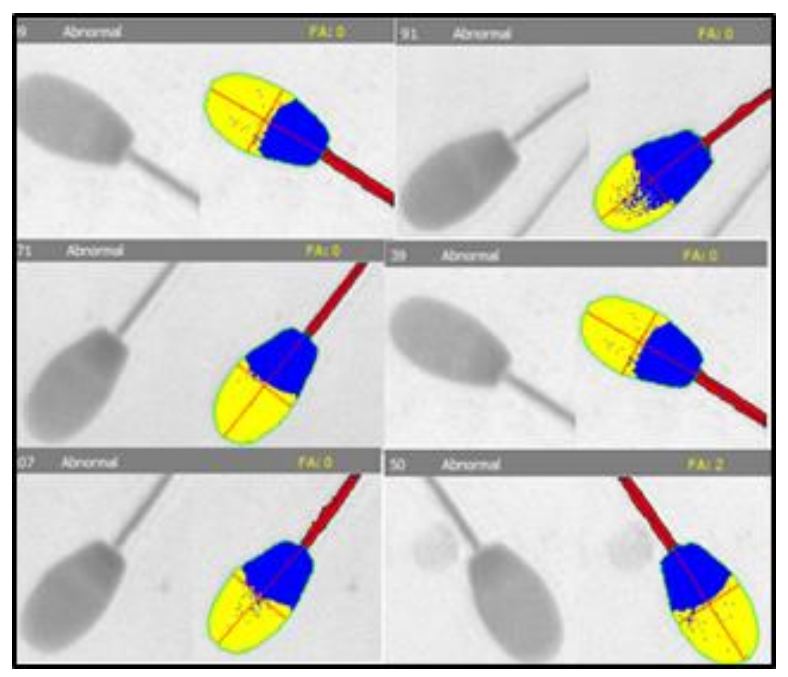

Figura 1. Espermatozoides de carnero. Imágenes de la izquierda capturadas en el microscopio, derecha imágenes digitalizadas: amarillo, acrosoma; azul, sección post acrosomal (área de la cabeza es la suma de ambas secciones); rojo, pieza intermedia. $44.05 \pm 2.69 \%$. Siendo estos valores como indicador de buena 
Estas diferencias no las encontramos en las variables no dimensionales de la cabeza como son la elipticidad, rugosidad elongación y regularidad y tampoco en las medidas de la pieza intermedia del espermatozoide.

\section{DISCUSIÓN}

En el trabajo de Perez et al. (2011) en condiciones Chuquibambilla - Puno, encontraron valores similares de volumen, y superiores de concentración, en relación con esta última variable las diferencias se deberían a la metodología de evaluación, de un sistema tradicional con uno CASA. Delgado (2013) en condiciones de Lima, reportó valores similares de volumen y motilidad, superiores de concentración e inferiores de integridad de la membrana, en este caso las diferencias serían por el medio ambiente y también por el sistema de evaluación de las variables microscópicas. Cabrera et al. (2011) en Lima, reporta valores similares de viabilidad e integridad de la membrana, y superiores de volumen, concentración espermática y motilidad, las diferencias se deberían a la raza de los animales, medioambiente y sistema de evaluación de las variables microscópicas.

Los parámetros morfométricos de la cabeza del espermatozoide (longitud, anchura, área y perímetro), en fresco son similares a los reportados por Maroto-Morales et al. (2010) y Bravo (2010), aunque este último señala que existen diferencias en los tamaños de las cabezas, mostrando un comportamiento estacional con valores más elevados en otoño y a principios de invierno en el hemisferio norte. Martí et al. (2011) evaluaron los cambios en la morfometría de la cabeza del espermatozoide de carneros los que fueron criopreservados en relación con la edad de madurez sexual, encontró evaluar el efecto de la congelación en los parámetros morfométricos de los espermatozoides del carnero que éstos son mayores en animales jóvenes, pero en general los valores que ha obtenido son inferiores a los reportados por Maroto-Morales et al. (2010), Bravo (2010). Algunas diferencias con nuestro trabajo, debido podrían deberse al manejo imágenes con diferentes sistemas (MOTIC®), ISAS, Proiser), técnicas de tinción (Hemacolor ${ }^{\circledR}$, Hematoxilina de Harris).

En el presente trabajo encontramos que tanto el área como el perímetro del espermatozoide de carnero criopreservado, es significativamente menor $(p<0.05)$, en relación a sus dimensiones en fresco y refrigerado, lo cual también ha sido comprobado por Marco-Jiménez et al. (2006) en espermatozoides de anguilas y Ramón et al. (2013) en ovinos, que podría deberse a diferentes razones que incluyen los cambios osmóticos, las alteraciones en algunos compartimientos de las células, sobrecondensación de la cromatina nuclear y daño o pérdida del acrosoma (Maroto-Morales et al., 2016). En relación con el acrosoma en el presente trabajo, se ha encontrado una disminución significativa $(p<0.05)$ del $8.7 \%$ en el porcentaje del acrosoma con respecto a espermatozoide de semen fresco, apoyando a los reportes Maroto-Morales et al. (2016).

\section{CONCLUSIÓN}

Nuestros resultados indican que algunos parámetros morfométricos del espermatozoide (longitud, anchura, área y perímetro de la cabeza) criopreservado son de menor dimensión $(p<0.05)$ a lo encontrado en semen fresco y refrigerado.

\section{Agradecimientos}

Agradecemos al Laboratorio de Biotecnologías Reproductivas de la Escuela Profesional de Zootecnia en el Centro Experimental La Raya, por las facilidades brindadas para la ejecución de la presente investigación, así como al proyecto canon Estudio, Validación y Aplicación de Biotecnologías Reproductivas en el Proceso de Mejoramiento y Valoración.

Genética en Alpacas en las Provincias de Canas, Canchis y Quispicanchi en la Región Cusco, por las facilidades en reactivos y material de laboratorio.

\section{CONFLICTO DE INTERESES}

Los autores firmantes del presente trabajo de investigación declaran no tener ningún potencial conflicto de interés personal o económico con otras personas $u$ organizaciones que puedan influir indebidamente con el presente manuscrito.

\section{CONTRIBUCIONES DE LOS AUTORES}

Preparación y ejecución: $\mathrm{HC}, \mathrm{WCh}, \mathrm{HQ}$

Desarrollo de la metodología: WCh, $\mathrm{HQ}$

Concepción y diseño: $\mathrm{HC}, \mathrm{CO}$

Edición del artículo: $\mathrm{HC}, \mathrm{WCh}, \mathrm{CO}$

Supervisión del estudio: $\mathrm{HC}, \mathrm{CO}$

\section{REFERENCIAS}

- Aguilar G, Amaro K, Hernández G. Evaluación de dos diluyentes para la conservación de semen de ovino: "yema de huevo vs lecitina de soya". Trabajo de investigación modular. Universidad Autónoma Metropolitana. Xochimilco. México. 2013

- Bravo J. Estudio de la influencia de la estación sobre los parámetros seminales y morfométricos del espermatozoide de morueco de la raza lle de France. Tesis Facultad de Veterinaria. Universidad de Extremadura. Cáceres. España. 2010.

- Cabrera P, Ayulo A, Pantoja C. Efecto del dilutor TRIS y citrato con yema de huevo de codorniz sobre la viabilidad espermática en semen ovino congelado en pajillas. Rev Inv Vet Perú. 201 1; 22(2): 105-113

- Delgado C. Evaluación espermática de semen de ovino tratado por la técnica de gradiente de densidad. Tesis Escuela Académico Profesional de Biología. Universidad Ricardo Palma. Lima. Perú. 2013.

- Gomedio M, Roldan ERS. Implications of diversity in sperm size and function for sperm competition and fertility. Int. J Dev Biol. 2008; 52 (5-6): 439-447

- Gravance C, White C, Robertson K, Champion Z, Casey P. The effects of cryopreservation on the morphometric dimensions of caprine sperm heads. Anim. Reprod. Sci. 1997; 49 (1): 37-43.

- Hafez ESE, Hafez B. Reproducción e inseminación artificial en animales. McGraw-Hill Inteamericana. México DF. 2004. $512 \mathrm{pp}$.

- Marco-Jiménez F, Garzón D, Peñaranda D, Pérez L, Viudes de Castro M, Vicente J, Asturiano J. Cryopreservation of European eel (Anguilla anguilla) spermatozoa: effect of dilution ratio, foetal bovine serum supplementation and cryoprotectants. Cryobiology. 2006; 53 (1): 51 -57.

- Maroto A. Evaluación objetiva de la morfometría de los espermatozoides de ovino (Ovis aries). Relaciones con la fertilidad. Tesis Doctoral. Universidad de Castilla-La Mancha. Instituto de Investigación en Recursos Cinegéticos. Albacete. España. 2012 
- Maroto-Morales A, Ramón M, García-Álvarez O, Soler A, Esteso M, Martínez F, Pérez M, Garde J. Characterization of ram (Ovis aries) sperm head morphometry using the Sperm -Class Analyzer. Theriogenology. 2010; 73 (4):437-448.

- Maroto-Morales A, García-Álvarez O, Ramón M, MartínezPastor F, Fernández-Santos M, Soler A, Garde. Current status and potencial of morphometric sperm analysis. J. Asian Journal of Andrology. 2016; 18 (6): 863-870.

- Martí J, Aparicio I, García-Herreros M. Head morphometric changes in cryopreserved ram spermatozoa are related to sexual maturity. Theriogenology. 2011 ; 75 (3): 473-481

- Núñez-Martinez I, Moran JM, Peña FJ. Do computer-assisted, morphometric - derived sperm characteristics reflects DNA status in canine spermatozoa? Reprod. Domest Anim. 2005; 40 (6): 537 - 543

- Perez U, Perez M, Mellisho E. Viabilidad espermática en semen de carnero congelado por dos métodos. SPERMOVA. 2011 (1); 1: 127 - 128.

- Ramón $M$, Pérez-Guzmán $M D$, Jiménez-Rabadán $P$, Esteso MC, García-Alvarez O, Maroto A, Anel-López L, Soler A, Fernández R, Garde J. Sperm cell population dynamics in ram semen during the cryopreservation process. PLoS ONE. 2013; 8 (3): e59189

- Verstegen J, Iguer-Ouada M, Onclin K. Computer assisted semen analyzers in andrology research and veterinary practice. Theriogenology. 2002; 57(1): 149-179. 